\title{
Natural Language Semantics and Its Computable Analysis $^{\star}$
}

\author{
Zhao Liang ${ }^{186-15011578662]}$ and Zou Chongli ${ }^{2[86-010-87896823]}$ \\ 1 Tianjin University of Finance and Economics, Tianjin 300222, China \\ zlcass@163.com \\ 2 Chinese Academy of Social Sciences, Beijing 100005, China \\ chlizou@263.net
}

\begin{abstract}
With an illustrative example of $5 \times 3=5 \times 3$, the first section explained the major problem in the natural language interpretation process. The second section introduced Frege's famous idea of 'sense'. And this paper argues that Frege's definition is not constructive. The Third section contributes a subtle amendment to the syntax of IL (Intentional Logic). Traditionally, the three basic types $e, t$ and $s$ were not treated as a part of vocabulary of IL, while this paper argues that this treatment has allowed a much explicit way to coding programs in real algorithm. Given this treatment, "an expression $\alpha$ of type $\tau$ " is shortened to " $\alpha \mid \tau$ ". In the end of section three, this paper gave a natural language sentence to illustrate the intention and extension operators. And furthermore, there comes the analyses of information preservation ability of these operators.
\end{abstract}

Keywords: sense $\cdot$ reference $\cdot$ meaning $\cdot$ intention $\cdot$ extension.

\section{1 introduction}

The concept of artificial intelligence has been around ever since the advent of computer. While it is not until GPU is exploited in the deep learning algorithm at the beginning of the 21st century, did study on artificial intelligence boomed like an overnight sensation. Thanks to the development of computer science and engineering, even private laptops are quite efficient to run a variety of artificial intelligence algorithms on them. On the other hand, there is a treasure trove of information stored on the internet publicly to feed the data-starved learning machines all over the globe. With the right tool and limitless materials, the prosperity of artificial intelligence cannot be failed.

It seems that artificial intelligence serves as the Holy Grail of almost everything, but it is actually not. Artificial intelligence is catching up with human beings in more ways than one, especially in pattern recognition of voices, faces, handwritings etc. The best AI does even better than the best human board game master. But even the smartest machine can barely understand human language properly. Human language, also known as natural language, is a promising candidate for the last citadel

\footnotetext{
^ Supported by The National Social Science Fund of China.
} 
of Homo sapiens' half ludicrous, half pathetic sense of superiority over machine. Speaking a foreign language is seemingly not so hard as becoming a chess master, and speaking mother language is even much easier and more natural, which is the name, natural language, came after. While nine-year-old children could regale themselves with tall tales, the bedtime stories still do not make much sense to the best AI. Natural language processing with statistical methods and machine learning algorithms is a successful part of artificial intelligence, whereas there are inherent defects in this magic touch. The crux of the problem resides in the interpretation of expressions, which statistical methods alone do not help so much, because natural language is intentional rather than extensional.

Mathematics is the absolute epitome of extensional language. $5 \times 3=3 \times 5$ is an expression declares the equality between two terms. This expression hold true, because both $5 \times 3$ and $3 \times 5$ refers to the same number 15 . However, for a five years old boy, he has three apples in five bags each, and he is sure all these apples is all these apples, that is $15=15$, but he may not sure how many larger bags will do to take all these apples with five apples each. He might be surprised that three larger bags will just do to take all these apples, because $5 \times 3=3 \times 5$ update his knowledge with new information. This boy knows that all these apples are all these apples, that is $5 \times 3=5 \times 3$, and both $5 \times 3$ and $3 \times 5$ refer to the same number 15 , thus $5 \times 3=3 \times 5$ contribute nothing new information to $5 \times 3=5 \times 3$. The question is that where the new information came from that updated the boy's knowledge. It is the meaning of $5 \times 3$ that differs from the meaning of $3 \times 5$, which in the knowledge context that gave rise to the new information. The context where the equality hold true, whenever the replacement of same reference happens, is an extensional context. The context where equality failed by replacement of expressions with same reference is an intentional context. In most cases, natural language is applied in an intentional context. As long as intentional context cannot be took into account properly, artificial intelligence will stay artificial retarded.

\section{The Meaning of Natural Language}

This $5 \times 3=3 \times 5$ is essentially raised the question of what identity should be treated in the context of cognition. The seminal work of Frege(1948) [1] preferred the item$\mathrm{s}$ on both sides of the equal sign treated as names or signs of objects rather than the object itself. In programming language, $C$ for example, there is an obvious difference between the assignment operator "=" and the equality operator "=="[2]. But the assignment versus equality issue is quite another different story. Practically both assignment and equality refer to the items as the objects themselves. In the statement of " $\mathrm{a}=\mathrm{b}$ ", $\mathrm{a}$ is assigned the same information stored on the storage devices as $\mathrm{b}$ has already designated, but not the other way round. If $a$ and $b$ has already labeled the same information stored on the storage devices, " $a=b$ " could be removed from the code without messing up the programming anyway. And "a == b" is only served as the condition checking of the fact that $a$ and $b$ has already labeled the same information on the storage devices. Both the assignment operator " $="$ and the equality operator "==" has nothing to do with the cognitive significance in the case of natural 
language which was so-called Frege's Puzzles as in the case of $5 \times 3=3 \times 5$. Actually the Frege's Puzzle raises everywhere in natural language:

- Mark Twain is Samuel Clemens.

- Rachel is Bill's sister.

- Beijing is the capital of China.

Historically, the most famous example is the following two sentences[3]:

S1: The Morning Star is the Morning Star.

S2: The Morning Star is the Evening Star.

The ancient Greeks noticed 'two' bright stars in the sky at different times, which turned out to be the same same star we now known as planet Venus. Both The Morning Star and the Evening Star refers to Venus. If the meaning of a word is all about its reference, the two sentences reduce to one:

Venus is Venus.

While it is obvious that S2 giving more information than S1. Anyone without any astronomical knowledge could agree with S1. It should be noted that to agree with S2 one does not have to know both The Morning Star and the Evening Star refers to planet Venus, they just need the confidence of the fact that the two stars refers to the same thing. While reference is important to meaning, there is more than that. There comes the 'sense' that Frege used to describe the meaning of a word other than its reference. "the Morning Star" and "the Evening Star" both refer to the same thing, while they do have different senses. When it comes to the equation $5 \times 3=3 \times 5$, the two different senses are quite obvious: the sense of $5 \times 3$ is 'three times five', and the sense of $3 \times 5$ is 'five times three'.

Thanks to Frege's remarkable insights, it is much clear that there is something in the meaning which is quite different from its reference. However, Frenge's definition of sense is not constructive. His main idea is that there are two parts of meaning, and the sense is the rest part of meaning that the reference part can not help.

$$
\text { SENSE }=\text { MEANING }- \text { REFERENCE }
$$

'Meaning' is an umbrella term, which contains more than sense and reference. A sentence could be uttered more than once. Any particular utterance may have its own programmatic importance. It should be noted that all the programmatic role of 'Meaning' is beyond this paper. The 'meaning' discussed in this paper has nothing to do with emotion and imperative motives in any particular utterance of a sentence.

\section{The Intensional Logic}

Intensional logic is one of the most promising schemes to define sense constructively. The syntax of Intensional logic is practically Propositional calculus alongside the type theory part[4]. 
IL (Intensional Logic) is a Lambda Calculus with constants. The semantics of IL is practically an embedded part of IL which cannot be changed and no need to change. Every syntactic well formed expression is fixed with a semantic interpretation. The IL here will be furnished with a modal operator and two tense operators. The intension of an expression $\alpha$ will be interpreted in terms of possible worlds and possible times[5].

\section{Definiton 1 The vocabulary of IL}

(1) An countable infinite set $\mathbf{V A R}=v_{1}, v_{2}, v_{3}, v_{4}, \cdots$

(2) An nonempty set CON

(3) $e, t, s$

(4) $\neg, \vee$

(5) $\forall$

(6) $(,\langle|)$,

(7) $\lambda x$, for every $x \in$ VAR

It should be noticed that " $\lambda x$ " as a whole is a symbol in the vocabulary of IL. The $x$ in " $\lambda x$ " can never be treated as a variable in VAR. Actually it is much less misleading to use " $\lambda_{x}$ " instead of " $\lambda x$ ". The nonempty set CON is the set of all constants. When possible worlds and possible times are concerned CON are bound to be uncountably infinite simply because the moments on the time line is as uncountably infinite much as the real numbers.

\section{Definiton 2 The type part of an expression of IL}

$T P$ refers to the set of all type parts. And then a member of set $T P$ can be derived by applying the following rules finitely:

(1) $e \in T P$, and $t \in T P$

(2) If $\tau_{1} \in T P$, and $\tau_{2} \in T P$, then $\left\langle\tau_{1}, \tau_{2}\right\rangle \in T P$

(3) If $\tau \in T P$, then $\langle s, \tau\rangle \in T P$

\section{Definiton 3 The expression of IL}

$W E$ refers to the set of all well-formed expressions. Then a member of set $W E$ can be obtained by applying the following rules finitely:

(1) If $a \in T P$, then $v \mid a$ and $c \mid a$ are expressions, where $v \in V$ and $c \in \mathscr{C}$.

(2) If $\alpha \mid\left\langle\tau_{1}, \tau_{2}\right\rangle$ and $\beta \mid \tau_{1}$ are expressions, then $(\alpha \beta) \mid \tau_{2}$ is an expression.

(3) If $\alpha \mid \tau_{1}$ is an expression and $x \mid \tau_{2}$ is an variable, $\lambda x \alpha \mid\left\langle\tau_{2}, \tau_{1}\right\rangle$ is an expression.

(4) If $\phi \mid t$ and $\psi \mid t$ are expressions, then so are $(\neg \phi) \mid t$ and $(\phi \vee \psi) \mid t$.

(5) If $\phi \mid t$, and $x \mid \tau$ are expressions, where $x$ is a variable, then $\forall x \phi \mid t$ is an expression.

(6) If $\alpha \mid \tau_{1}$ and $\beta \mid \tau_{2}$ are expressions, then $(\alpha=\beta) \mid t$ is an expression.

(7) If $\phi \mid t$ is an expression, then $\square \phi \mid t$ is an expression.

(8) If $\phi \mid t$ is an expression, then $\mathbf{F} \phi \mid t$ is an expression.

(9) If $\phi \mid t$ is an expression, then $\mathbf{P} \phi \mid t$ is an expression.

(10) If $\alpha \mid \tau$ is an expression, then ${ }^{\wedge} \alpha \mid\langle s, \tau\rangle$ is an expression. 
(11) If $\alpha \mid\langle s, \tau\rangle$ is an expression, then ${ }^{\vee} \alpha \mid \tau$ is an expression

Then " $\alpha \mid \tau \in W E^{\prime \prime}$ means " $\alpha \mid \tau$ is an expression". $\tau$ is the type part of the expression $\alpha \mid \tau$, and $\alpha$ the expression part of the expression $\alpha \mid \tau$. The last two rules introduce the intentional operator and extensional operator respectively. ${ }^{\wedge} \alpha \mid\langle s, \tau\rangle$ could serve as the intention of $\alpha$, and $\alpha \mid\langle s, \tau\rangle$ the extension of $\alpha$.

$W E_{\tau}$ refers to the set of all the expressions with type part $\tau . V A R_{\tau}$ refers to the set of all the expressions with expression part as a variable and type part as $\tau$. $C O N_{\tau}$ refers to the set of all the expressions with expression part as a constant and type part as $\tau$.

The semantics of IL could simply be defined step by step along with the reductive definition of syntax. The first step is to built the domain $D_{\tau}$ of every type $\tau$. Next step is to specify all the constants with valuation function $f$. Finally for any assignment function $g$ there is an interpretation function \|\|$^{g}$ which interpreted all the wellformed expressions of IL.

Epistemology is the fancy name for the theory of knowledge, which gave rise to a whole bunch of intentional contexts. And epistemic logic is the logic of knowledge, which could be treated as a part of Epistemology. While initially the study of intentional logic begins with modal logic rather than epistemic logic. And Kripke semantics still plays a major role in the intentional logic. That's because the intention and extension of an expression can be treated as two operators to form new expressions ${ }^{\wedge} \alpha$ and ${ }^{\vee} \alpha$ respectively, where the explanation of an intentional expression is modeled in Kripke's possible-world logic. The same expression could be interpreted into different means in different conditions. This ingenious idea is largely credited to Montagueą́ŕs work, and Morrill(2011)[6] "gave a technical refinement in this idea".

The building blocks of semantics of IL are four non-empty sets:

\begin{tabular}{l|l} 
individuals & $A$ \\
\hline truth values & $\{0,1\}$ \\
\hline possible worlds & $W$ \\
\hline possible times & $T$
\end{tabular}

With these original building blocks it is possible to build any domain $D_{\tau}$ with type $\tau$ by recursive definition on types.

\begin{tabular}{c|l} 
type & domain \\
\hline \hline$t$ & $D_{t}=\{0,1\}$ \\
\hline$e$ & $D_{e}=A$ \\
\hline$\left\langle\tau_{1}, \tau_{2}\right\rangle$ & $D_{\left\langle\tau_{1}, \tau_{2}\right\rangle}=D_{\tau_{2}}^{D_{\tau_{1}}}$ \\
\hline$\langle s, \tau\rangle$ & $D_{\langle s, \tau\rangle}=D_{\tau}^{W \times T}$
\end{tabular}

Let the model is set with $A,\{0,1\}, W, T$, and the universal valuation function $f$. Given any universal assignment function $g$, and then it is all set to interpret every well-formed expressions of IL. Interpretation function \|\|$^{w, t, g}$ is defined recursively over expressions. The routine procedure given as follows[6]: 
(1) If $c \in C O N$, then $\|c\|^{w, t, g}=f(c)(\langle w, t\rangle)$

It should be noticed that the interpretation of a constant has nothing to do with the assignment function $g$.

(2) If $x \in V A R$, then $\|x\|^{w, t, g}=g(x)$

It should be noticed that the interpretation of a variable has nothing to do with the spacetime $\langle w, t\rangle$.

(3) If $\alpha \mid\left\langle\tau_{1}, \tau_{2}\right\rangle$ and $\beta \mid \tau_{1}$, then $\|(\alpha \beta)\|^{w, t, g}=\|\alpha\|^{w, t, g}\left(\|\beta\|^{w, t, g}\right)$

(4) If $\alpha \mid \tau_{1}$ and $x \mid \tau_{2},\|\lambda x \alpha\|^{w, t, g} \in D_{\tau_{1}}^{D_{\tau_{2}}}$, and $m \in D_{\tau_{2}}$, then $\|\lambda x \alpha\|^{w, t, g}(m) \in D_{\tau_{1}}$, such that

$$
\|\lambda x \alpha\|^{w, t, g}(m)=\|\alpha\|^{w, t, g^{[x \rightarrow m]}}
$$

where

$$
g^{[x \rightarrow m]}(y)= \begin{cases}g(y) & y \neq x \\ m & y=x\end{cases}
$$

(5) If $\phi \mid t$, and then

$$
\|\neg \phi\|^{w, t, g}=\left\{\begin{array}{l}
1\|\phi\|^{w, t, g}=0 \\
0\|\phi\|^{w, t, g}=1
\end{array}\right.
$$

(6) If $\phi \mid t$ and $\psi \mid t$, then

$$
\|(\phi \vee \psi)\|^{w, t, g}=\left\{\begin{array}{l}
1 \text { else } \\
0\|\phi\|^{w, t, g}=0 \text { and }\|\psi\|^{w, t, g}=0
\end{array}\right.
$$

(7) If $\alpha \mid \tau$ and $\beta \mid \tau$, then $(\alpha=\beta) \mid t$ such that

$$
\|(\alpha=\beta)\|^{w, t, g}=\left\{\begin{array}{l}
1\|\alpha\|^{w, t, g}=\|\beta\|^{w, t, g} \\
0 \text { else }
\end{array}\right.
$$

(8) If $\phi \mid t$, and $x \mid \tau$, where $x$ is a variable, then

$$
\|\forall x \phi\|^{w, t, g}=\left\{\begin{array}{l}
1 \text { else } \\
0 \exists m \in D_{\tau} \text { such that }\|\phi\|^{w, t, g^{[x \rightarrow m]}}=0
\end{array}\right.
$$

(9) If $\phi \mid t$, then

$$
\|\square \phi\|^{w, t, g}=\left\{\begin{array}{l}
1 \text { for all }\left\langle w^{\prime}, t^{\prime}\right\rangle \in W \times T \text { such that }\|\phi\|^{w^{\prime}, t^{\prime}, g}=1 \\
0 \text { else }
\end{array}\right.
$$

(10) If $\phi \mid t$, then

$$
\|\mathbf{F} \phi\|^{w, t, g}=\left\{\begin{array}{l}
1 \quad \exists t^{\prime}>t \text { such that }\|\mathbf{F} \phi\|^{w, t^{\prime}, g}=1 \\
0 \text { else }
\end{array}\right.
$$

(11) If $\phi \mid t$, then

$$
\|\mathbf{P} \phi\|^{w, t, g}=\left\{\begin{array}{l}
1 \exists t^{\prime}<t \text { such that }\|\mathbf{P} \phi\|^{w, t^{\prime}, g}=1 \\
0 \text { else }
\end{array}\right.
$$


(12) If $\alpha \mid \tau$, then $\left\|^{\wedge} \alpha\right\|^{w, t, g} \in D_{\tau}^{W \times T}$ such that

$$
\left\|^{\wedge} \alpha\right\|^{w, t, g}\left(\left\langle w^{\prime}, t^{\prime}\right\rangle\right)=\|\alpha\|^{w^{\prime}, t^{\prime}, g}
$$

(13) If $\alpha \mid\langle s, \tau\rangle$, then $\left\|^{\vee} \alpha\right\|^{w, t, g} \in D_{\tau}$ such that

$$
\left\|^{\vee} \alpha\right\|^{w, t, g}=\|\alpha\|^{w, t, g}(\langle w, t\rangle)
$$

The last two items interpret the intentional operator and extensional operator respectively. ${ }^{\wedge} \alpha$ refers to the "intention" of $\alpha$, and ${ }^{\vee} \alpha$ the "extension" of $\alpha$. It shows that the interpretation of ${ }^{\wedge} \alpha$ has nothing to do with the spacetime $\langle w, t\rangle$. In other words, the interpretation of ${ }^{\wedge} \alpha$ is always the same regardless of possible worlds and times. And the interpretation of ${ }^{\wedge} \alpha$ is a function defined on $W \times T$, which practically multiplied all the spacetimes in each and every spacetime. Initially, suppose there were $n$ spacetimes in the model, whenever "intention" is concerned it is actually $n^{2}$ spacetimes in picture. The interpretation of ${ }^{\vee} \alpha$ has simply the opposite effect, such that only each of the spacetime $\langle w, t\rangle$ in spacetime $\langle w, t\rangle$ is left.

It is convenient to go back to the simplified model to illustrate the major point. Let $W=\left\{w_{1}, w_{2}\right\}$ and $T=\left\{t_{1}, t_{2}\right\}$. And then there are only four spacetimes in this model:

$$
\frac{\left(w_{1}, t_{2}\right) \mid\left(w_{1}, t_{1}\right)}{\left(w_{2}, t_{1}\right) \mid\left(w_{2}, t_{2}\right)}
$$

Actually, any specific wold or time is not concerned. The four different spacetimes could simply be treated as four possible worlds. And then the model is $\langle A,\{0,1\}, W, f\rangle$, where $W=\left\{w_{1}, w_{2}, w_{3}, w_{4}\right\}$ :

$$
\frac{w_{2} \mid w_{1}}{w_{3} \mid w_{4}}
$$

Let $\|\alpha\|^{w_{1}, t_{1}, g}=x_{1} ;\|\alpha\|^{w_{1}, t_{2}, g}=x_{2} ;\|\alpha\|^{w_{2}, t_{1}, g}=x_{3} ;\|\alpha\|^{w_{2}, t_{2}, g}=x_{4}$, and then the interpretation of $\alpha$ could be illustrated as

$$
\begin{array}{l|l}
x_{2} & x_{1} \\
\hline x_{3} & x_{4}
\end{array}
$$

Then the interpretation of ${ }^{\wedge} \alpha$ :

$$
\begin{array}{l|l||l|l}
x_{2} & x_{1} & x_{2} & x_{1} \\
\hline x_{3} & x_{4} & x_{3} & x_{4} \\
\hline \hline x_{2} & x_{1} & x_{2} & x_{1} \\
\hline x_{3} & x_{4} & x_{3} & x_{4}
\end{array}
$$

The process from ${ }^{\wedge} \alpha$ to ${ }^{\vee \wedge} \alpha$ :

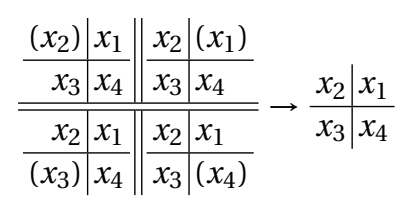


As a matter of fact it has illustrated $\left\|^{\vee \wedge} \alpha\right\|^{w, t, g}=\|\alpha\|^{w, t, g}$ in a small-scale case.

It is much convenient and intuitive to illustrate this counterexample in diagram$\mathrm{s}$ which introduced in the end of section 2.3. This counterexample is an simplified model with only four spacetimes which could be labeled with $A, B, C$ and $D$ :

$$
A=\left\langle w_{1}, t_{1}\right\rangle, B=\left\langle w_{1}, t_{2}\right\rangle, C=\left\langle w_{2}, t_{1}\right\rangle, D=\left\langle w_{2}, t_{2}\right\rangle
$$

And then the diagram could looked like this

$$
\begin{array}{l|l}
B & A \\
\hline C \mid D
\end{array}
$$

Suppose $A, B, C$ and $D$ are four different countries with different currencies. The following sentence could simulate the expression in the counterexample.

\section{currency has purchasing power}

In different countries the "currency" refers to different currencies. For instance, in country $A$ the "currency" refers to "currency of country $A$ ". It is true that currency of country $A$ has purchasing power in country $A$, while it is false in country $B, C$ and $D$. A fat stack of cash of country $A$ does you no good in country $B, C$ and $D$. This interpretation of "currency" is only the case of country $A$ :

$$
\begin{array}{l|l}
0 & 1 \\
\hline 0 & 0
\end{array}
$$

If the interpretation of "currency" in country $B, C$ and $D$ is included, the bigger picture looks like this:

$$
\begin{array}{l|l||l|l}
1 & 0 & 0 & 1 \\
\hline 0 & 0 & 0 & 0 \\
\hline \hline 0 & 0 & 0 & 0 \\
\hline 1 & 0 & 0 & 1
\end{array}
$$

As sentence "currency has purchasing power" simulates the expression $\alpha$, the following diagram could illustrate the application of extensional operator and intentional operator successively:

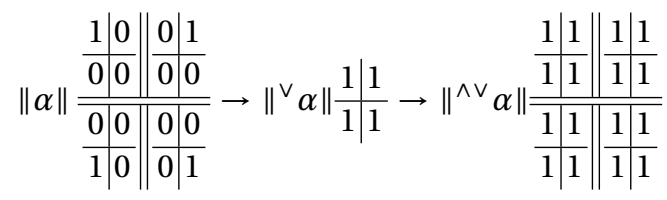

It is clear that the interpretation of ${ }^{\wedge} \vee \alpha$ is quite different from the interpretation of $\alpha$. Extensional operator was applied first, and then the interpretation of ${ }^{\vee} \alpha$ shows that it is true in all the four countries. And the sentence "currency has purchasing power" does seem like a plain truth. However, the truth is not the whole truth. It is essentially lost some information in the extensional interpretation, and the following 
intentional interpretation could not restore it. The lost information is just the intention of the sentence, i.e. the currency of a particular country has purchasing power in this particular country. The intentional interpretation practically multiplied the whole piece of information faithfully in every spacetimes, and thus immuned to extensional interpretation once. Thus the interpretation of ${ }^{\vee \wedge} \alpha$ was expected to be just the same as the interpretation of $\alpha$.

\section{Conclusion}

The introduction part explains the crux of the problem in natural language processing. The example of $5 \times 3=5 \times 3$ takes most of the credit to illustrate this problem. With this illustrative example this paper has clarified the distinction between the assignment operator "=" and the equality operator "==" has nothing to with the cognitive significance of sense. The Third section deals with syntax and sematic of IL. It has showed that the three basic types $e, t$ and $s$ could be included in the vocabulary of IL. The result is that "an expression $\alpha$ of type $\tau$ " [7] is shortened to expression " $\alpha \mid \tau$ ", where $\alpha$ is the expression part and $\tau$ the type part. And then the following recursive definitions became explicit and economic. This amendment has allowed intentional logic much explicit to coding programs in real algorithm. In the end of section three, this paper gave an natural language sentence to illustrate the intention and extension operators. And it shows that intentional interpretation preserved the whole piece of information in the meaning, whereas extensional interpretation are bound to lose some. In this sense Intentional Logic is not only an expansion of Extensional Logic, but also enjoys much power of interpretation.

\section{References}

1. Frege, G. (1948). Sense and reference. The philosophical review, 57(3), 209-230.

2. Damas, Luis. Type assignment in programming languages.The University of Edinburgh, 1984.

3. Kratzer, Angelika, and Irene Heim. Semantics in generative grammar. Vol. 1185. Oxford: Blackwell, 1998.

4. L.Zhao, C.L.Zou(2018). Intentional Logic as a Possible Scheme to Sematics Computation,Journal of Chongqing University of Technology(Social Science) Vol.32 No.6 2018

5. Glyn V. Morrill, Type Logical Grammar,Kluwer Academic Publishers, The Netherlands, 1994.

6. Glyn V. Morrill, Categorial Grammar, Oxford Univ. Press, New York, 2011.

7. L.T.F. Gamut, Logic, Language, and Meanimg, The University of Chicago Press, Chiacago and London, 1991. 